\title{
Research Paper: Patient Safety Climate and Its Affecting FactorsAmong Rehabilitation Health Care Staff of Hospitals and Rehabilitation Centers in Iran-Tehran
}

\author{
Alireza Khammar ${ }^{1}$ (D), Mohsen Poursadeghiyan ${ }^{2,3}$ (D), Hossein Marioryad ${ }^{4}$ (D), Reza Nabi Amjad (D), Monir Alimohammadi $^{6}$ (D), \\ Mohammad Khandan ${ }^{7 *}$ (D) \\ 1. Department of Occupational Health Engineering, School of Health, Zabol University of Medical Sciences, Zabol, Iran. \\ 2. Health in Emergencies and Disasters Research Center, University of Social Welfare and Rehabilitation Sciences, Tehran, Iran. \\ 3. Department of Ergonomics, Pediatric Neurorehabilitation Research Center, University of Social Welfare and Rehabilitation Sciences, Tehran, Iran. \\ 4. Social Determinants of Health Research Center, Yasuj University of Medical Sciences, Yasuj, Iran. \\ 5. Non-Communicable Diseases Research Center, Alborz University of Medical Sciences, Karaj, Iran. \\ 6. Health Management and Economics Research Center, Iran University of Medical Sciences, Tehran, Iran. \\ 7. Department of Occupational Health Engineering, Faculty of Health, Qom University of Medical Sciences, Qom, Iran.
}

\begin{tabular}{|l|l|}
\hline $\begin{array}{c}\text { Use yur devic to scan } \\
\text { and read the article online }\end{array}$ & $\begin{array}{l}\text { Crtation Khammar A, Poursadeghiyan M, Marioryad H, Nabi Amjad R, Alimohammadi M, khandan M. Patient Safety Cli- } \\
\text { mate and Its Affecting Factors Among Rehabilitation Health Care Staff of Hospitals and Rehabilitation Centers in Iran-Tehran. } \\
\text { Iranian Rehabilitation Journal. 2019; 17(1):39-48. http://dx.doi.org/10.32598/irj.17.1.39 }\end{array}$ \\
\hline dol & http://dx.doi.org/10.32598/irj.17.1.39
\end{tabular}

\section{(i) (3)}

Article info:

Received: 17 Oct 2018

Accepted: 28 Jan 2019

Available Online: 01 Mar 2019

Keywords:

Patient safety climate, Safety culture, Safety attitude, Job satisfaction, Rehabilitation

\section{ABSTRACT}

Objectives: Hospitals and clinical centers are concerned about patient safety. Safety climate is a perceived value of safety in an organization that could improve the safety of workers and patients. The present research was conducted to study the safety climate of patients in the hospitals and rehabilitation centers affiliated to the University of Social Welfare and Rehabilitation Sciences.

Methods: This descriptive-analytical study was conducted on 300 nurses and nurse's aides (healthcare staff) who were selected by stratified sampling method, from two hospitals and three clinics, in 2017. Data collection tools included Patient Safety Climate Scale presented by Kudo and a demographic data questionnaire. The obtained data were analyzed by SPSS using descriptive statics like frequencies and percentages. Furthermore, Mann-Whitney U test and Kruskal-Wallis test were used to analyze the obtained data and compare the mean scores, respectively.

Results: The Mean \pm SD age and work experience of study participants were $36.7 \pm 6.79$ and $9.46 \pm 5.8$ years, respectively. The patient safety climate sub-factors were significantly different between males and females $(\mathrm{P}<0.05)$ except for reporting aspect $(\mathrm{P}>0.05)$. Patient safety climate was only different in nursing condition $(\mathrm{P}=0.013)$ among studied healthcare centers. Also, only fatigue reduction was different among various studied wards $(\mathrm{P}=0.035)$, where intensive care unit had the lowest score $(2.12 \pm 2.0)$.

Discussion: Overall, the poor condition of patient safety climate was found in the studied rehabilitation centers. Therefore, it is recommended to improve nurses' attitudes with the assistance of hospital managers, to enhance patient safety.

\section{* Corresponding Author:}




\section{Highlights}

- The patients' safety concerns are considered a top priority for the healthcare of rehabilitation centers.

- In studied rehabilitation centers, the patients' safety climate is only different with respect to nursing condition.

- The patients' safety climate is good in some areas such as supervisors' attitude, communication between nurses, communication with physicians, and reporting.

- Fatigue reduction, nursing condition, and opportunities for nursing education are not good enough to preserve the appropriate patients' safety climate.

\section{Plain Language Summary}

According to the World Health Organization, patient's safety refers to preventing healthcare accidental injuries. The main objective of healthcare systems with respect to patient's safety is to reduce the incidence and effects of associated complications and improve recovery from such injuries. Safety climate is one of the effective factors on patients' safety in organizations. There are several factors that affect safety climate. To conduct a safe environment for both patients and practitioners, it is critical to improve the assessment and attitudes on safety among different groups of people in healthcare settings. Considering the above-mentioned points, we conducted a study to evaluate the safety of the patients' climate in the hospitals and clinics affiliated to the University of Social Welfare and Rehabilitation Sciences. According to findings, all aspects of patients' safety climate were in appropriate condition. These aspects included feeling sleepy at work, enough time to rest, recreation, physical and mental fatigue in private life, lack of correlation between workload and the number of employees, and an opportunity to understand the patient's condition; other necessary items were also at a moderate level. The patients' safety climate in studied hospitals was not appropriate. Because safety climate is a major indicator of safety performance and recognizing its affecting factors is important, the assessment of this situation can be effective in reducing the incidence of medical errors.

\section{Introduction}

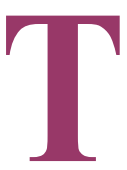

he patient safety concerns are considered as a top priority for healthcare organizations. The patient consequences in care or economic burden could be observed following safety incidents and clinical errors [1]. According to the World Health Organization, patient safety indicates preventing healthcare accidental injuries. The main objective of healthcare systems on patient safety is to reduce the incidence and effects of associated complications and improve recovery from such issues [1].

The occurrence rate of preventable mortality due to the inadequate measures of patient safety was over 97000 in the USA between 2005 and 2007 [2]. Safety climate is one of the effective factors on the safety of patients in organizations [3]. It is often considered as an indicator to determine patient safety because its changes like increased medical errors, can severely and directly affect patient's safety [1]. The staff perception of safety influence their motivation towards occupational safety behaviors [3].

Nurses are the main caregivers of the patients and responsible for many care activities; thus, they are strongly contributed to medical errors. Therefore, assessing nurses' attitudes and perceptions on safety may greatly impact the evaluation and safety climate level [4]. Patient safety climate in the hospital is a key element to increase patient safety, representing the perceived level of safety at a particular time and place [5].

Depression and stress are highly prevalent among nurses especially in rehabilitation and psychiatric settings where nurses provide care for patients with special conditions [6]. Stress has psychosomatics effects on all employers, including nurses [7-9]. Moreover, such psychological factors are correlated with safety climate and can affect patient safety [10]. Furthermore, the frequency of patient safety incidents is extremely high [11]. Therefore, it is necessary to explore healthcare staffs' attitudes about patient safety. Nurses may be aware of their es- 
sential role in providing safe care services and that they need to have positive safety attitudes [12].

To conduct a safe environment for both patients and practitioners, it is critical to improve the assessment and attitudes on safety among different groups of people in healthcare settings [13]. Considering the above-mentioned points, this study was conducted in Iran to evaluate the safety climate of patients in the hospitals and clinics affiliated to the University of Social Welfare and Rehabilitation Sciences.

\section{Methods}

This descriptive and analytical research was conducted on healthcare staff (nurses and nurse's aides) in Rofeideh Hospital and Razi Hospital and Nezam Mafi Clinic, Asma, and Akhavan affiliated to the University of Social Welfare and Rehabilitation Sciences, which were selected using stratified sampling method. The final sample size was estimated to be 300 persons. Finally, 57 participants from Rofeyide, 203 from Razi, 17 from Nezam mafi, 13 from Asma, and 10 participants from Akhavan clinic were selected.

The main inclusion criterion was being a nurse or nurse's aide. Respondents completed researcher-made demographic data questionnaire and Patient Safety Climate Scale (PSCS) developed by Kudo et al. [3].

The demographic data questionnaire consisted of age, gender, marital status, educational level, work experience, type of employment, the name of the hospital, and ward. PSCS included 30 items covering safety climate in 7 dimensions, as follows: opportunities for nursing education, communication between nurses, communication with physicians, fatigue reduction, superiors' attitudes, reporting, and nursing condition with responses in a 5-point Likert type scale format.

Data analyses were performed in respect of the obtained mean scores in each dimension, where the total score of each dimension was divided by the number of questions. Scores less than 2.6 indicated an unfavorable level, 2.6 to 4.3 moderate and greater than 4.3 , represented an ideal level. The content validity and reliability of the Persian version of the scale were confirmed and Cronbach's alpha of 0.832 was achieved as the internal reliability by previous research [14].

Results were collected and reported after approval by the Ethics Committee of University of Social Welfare and Rehabilitation Sciences. Informed consent forms were obtained from all nurses and nurse's aides; they were also informed that participating and leaving the study was voluntarily. All related information of the study participants were considered confidential.

Obtained data were analyzed using descriptive statistics like frequency and percentage by SPSS. KolmogorovSmirnov (K-S) test was applied to assess the normality of data. Then, Mann-Whitney U test and Kruskal-Wallis test were used to compare the mean scores.

\section{Results}

The Mean \pm SD scores of age and work experience were $36.7 \pm 6.79$ and $9.46 \pm 5.8$ years, respectively. The mean score of daily working time was 8.22 hours. In total, 169 (56.34\%) participants were female, and 249 (83\%) had a university degree. Moreover, 219 (73\%) subjects were officially employed. Also, 207 (69\%) participants were married. , Furthermore, 79 (26.33\%), 62 (20.67\%), 57 (19\%), 55 (18.33\%), and 47 (15.67\%) participants were selected from the internal, surgical, emergency, and pediatrics wards, respectively. Distribution of the Mean \pm SD scores of 7 dimensions of patient safety climate are presented in Table 1.

According to Table 1, all sub-factors of patient safety climate were in appropriate condition. These include feeling sleepy at work, enough time to rest, recreation, physical and mental fatigue in private life, the lack of correlation between workload and the number of employees, and an opportunity to understand the patient's condition; other items of requirements were also at moderate level.

According to the K-S test results, data were not normally distributed $(\mathrm{P}<0.05)$. Thus, non-parametric tests were applied. Distribution of the Mean \pm SD of the 7 dimensions of patient safety climate is listed in Table 2 according to the subjects' demographic characteristics.

\section{Discussion}

It is critical to recognize Nurses' attitude on the safety of patients in hospitals. Employees' perception on safety culture are correlated with their performance in clinical centers [15]; thus, the present study was conducted to measure such association. Numerous studies have investigated patient safety climate in general hospitals; however, few studies have addressed rehabilitation and psychiatric settings, in this regard. Therefore, this point can be considered as the novelty and importance of the present study. 
According to the obtained results, the level of patient safety climate was not appropriate in the hospitals. Although the study was against Baghaee et al. [16] research, it was similar to some other investigations [4]. Nurses especially in rehabilitation and psychiatric clinics, experience more unusual behaviors and violence by patients [17]. Singer et al. reported healthcare centers with better safety climate had a lower incidence of Patient Safety Indicators (PSIs); also, better safety climate among frontline personnel was associated with the lower risk of facing PSIs [18]. Therefore, lower levels of health outcomes will be expected in the studied organizations [19].

Healthcare organizations with stronger attitudes empower employees and provide psychological safety and comfort to take interpersonal risks, which enables people to prevent, solve, and learn more from problems at the frontlines of care delivery [11]. Mann-Whitney U test results suggested significant differences between males and females. This finding is in line with Vifladt et al. [20], but inconsistent with some others [4, 14].

Kruskal-Wallis test revealed no significant difference between employment status in terms of the mean scores of scale dimensions, except for the healthcare staff con- ditions $(\mathrm{P}=0.036)$. It means females had a better attitude on nursing conditions; in other words, females can better manage different conditions. In addition, nurses in the contract status of employment reported better nursing condition $(\mathrm{P}=0.034,2.93)$. Clinics (except for the clinics of two main hospitals of Rofeyide and Razi) had a better nursing condition in the viewpoints of studied nurses $(\mathrm{P}=0.013,3.1)$.

The obtained results indicated that men had more opportunities to educate in the nursing field (2.96 vs. 2.76). In our study, educational level had no variation effect on any factor $(\mathrm{P}>0.05)$. Brasaite et al. illustrated the effects of education on some aspects of patient safety climate like management perceptions but not the safety climate [12]. Females (mean score: 3.28) and males (mean score:3.6) had differences in communication with physicians $(\mathrm{P}=0.07)$. It means males can communicate better during nursing duties. Past researches reported that attitudes about patient safety was different among various job groups in hospitals [21].

In terms of wards, nurses working in the emergency department had the lowest mean score (2.34); likewise, Singer et al. reported that emergency department staff perceived lower levels of safety climate than other wards'

Table 1. Mean $\pm S D$ and distribution of patient safety climate items in terms of desirability

\begin{tabular}{|c|c|c|c|}
\hline Factor & Main Items & Mean $\pm S D$ & Desirability \\
\hline Superiors' attitudes & Courage to error reporting & $2 \pm 1.22$ & Moderate \\
\hline Superiors' attitudes & Authorities fair reaction in the event of a fault & $3.17 \pm 1.2$ & Moderate \\
\hline Superiors' attitudes & Proper guidance of supervisors & $3.34 \pm 1$ & Moderate \\
\hline Superiors' attitudes & Valuing the proposed staff about patient safety & $3.17 \pm 1.2$ & Moderate \\
\hline Relationships among nurses & Team work of healthcare staff & $3.44 \pm 1.11$ & Appropriate \\
\hline Relationships among nurses & Good relations between healthcare staff & $3.66 \pm 2$ & Appropriate \\
\hline Relationships among nurses & Staff cooperating with other sections when required & $3.46 \pm 0$ & Appropriate \\
\hline Communications with physicians & Proper guidance of nurse by physicians & $3.46 \pm 2$ & Appropriate \\
\hline Communications with physicians & $\begin{array}{l}\text { Good cooperation between healthcare staff and physi- } \\
\text { cians }\end{array}$ & $3.2 \pm 0$ & Moderate \\
\hline Communications with physicians & Easy communication with physicians about health issues & $3.24 \pm 2$ & Moderate \\
\hline Fatigue reduction & Lack of sleep & $2.50 \pm 1.10$ & Inappropriate \\
\hline Fatigue reduction & Enough time to relax & $2.42 \pm 2$ & Inappropriate \\
\hline
\end{tabular}




\begin{tabular}{|c|c|c|c|}
\hline Factor & Main Items & Mean $\pm S D$ & Desirability \\
\hline Fatigue reduction & Adequate opportunity for recreation & $2.23 \pm 0$ & Inappropriate \\
\hline Fatigue reduction & Not feeling mental fatigue & $2.40 \pm 2$ & Inappropriate \\
\hline Fatigue reduction & Not feeling physical fatigue & $2.35 \pm 1.70$ & Inappropriate \\
\hline Opportunities for nursing education & Appropriate training programs to improve job skills & $2.88 \pm 1.3$ & Moderate \\
\hline Opportunities for nursing education & Proper role in healthcare provision & $2.85 \pm 1.77$ & Moderate \\
\hline Opportunities for nursing education & Training programs for new healthcare staff & $2.77 \pm 2$ & Moderate \\
\hline Opportunities for nursing education & $\begin{array}{c}\text { Specific training programs tailored to the needs of each } \\
\text { individual }\end{array}$ & $2.7 \pm 1.67$ & Moderate \\
\hline Opportunities for nursing education & Improving the ability of employees & $2.72 \pm 2$ & Moderate \\
\hline Nursing conditions & Healthcare workers election based on ability & $2.93 \pm 1$ & Moderate \\
\hline Nursing conditions & Healthcare workers election based on clinical experience & $2.67 \pm 1.2$ & Moderate \\
\hline Nursing conditions & Sufficient number of health care worker staff & $2.35 \pm 1.13$ & Inappropriate \\
\hline Nursing conditions & $\begin{array}{l}\text { The appropriateness of work load and the number of } \\
\text { employees }\end{array}$ & $2.44 \pm 3$ & Inappropriate \\
\hline Nursing conditions & Sufficient time to understand the patient's condition & $2.56 \pm 0$ & Inappropriate \\
\hline Reporting & Positive change in reporting errors & $4.35 \pm 1.12$ & Moderate \\
\hline Reporting & Increased awareness of patient safety in reporting errors & $3.15 \pm 1.10$ & Moderate \\
\hline Reporting & Unpredictable nature of the error & $3.12 \pm 1.2$ & Moderate \\
\hline Reporting & Addressing medical errors & $2.84 \pm 0$ & Moderate \\
\hline Reporting & $\begin{array}{l}\text { Quick action by employees informed as soon as the error } \\
\text { occurred }\end{array}$ & $3.19 \pm 1.14$ & Moderate \\
\hline
\end{tabular}

Table 2. Level of the situation of the dimensions of patient safety climate based on average

\begin{tabular}{ccccc}
\hline \multirow{2}{*}{ Item } & Dimensions & \multicolumn{3}{c}{ Level of the Situation Based on Average } \\
\cline { 3 - 5 } & & Undesirable & Moderate & Good \\
\hline 1 & Supervisors' attitude & 26 & 26.6 & 46.4 \\
\hline 2 & Communication between nurses & 15 & 26 & 60 \\
\hline 3 & Communication with physicians & 23 & 31 & 45 \\
\hline 4 & Fatigue reduction & 66.6 & 15 & 17.6 \\
\hline 5 & Opportunities for nursing education & 43 & 31.6 & 24.4 \\
\hline 6 & Nursing condition & 60 & 24.4 & 16.6 \\
\hline 7 & Reporting & 30.4 & 33.6 & 35 \\
\hline
\end{tabular}




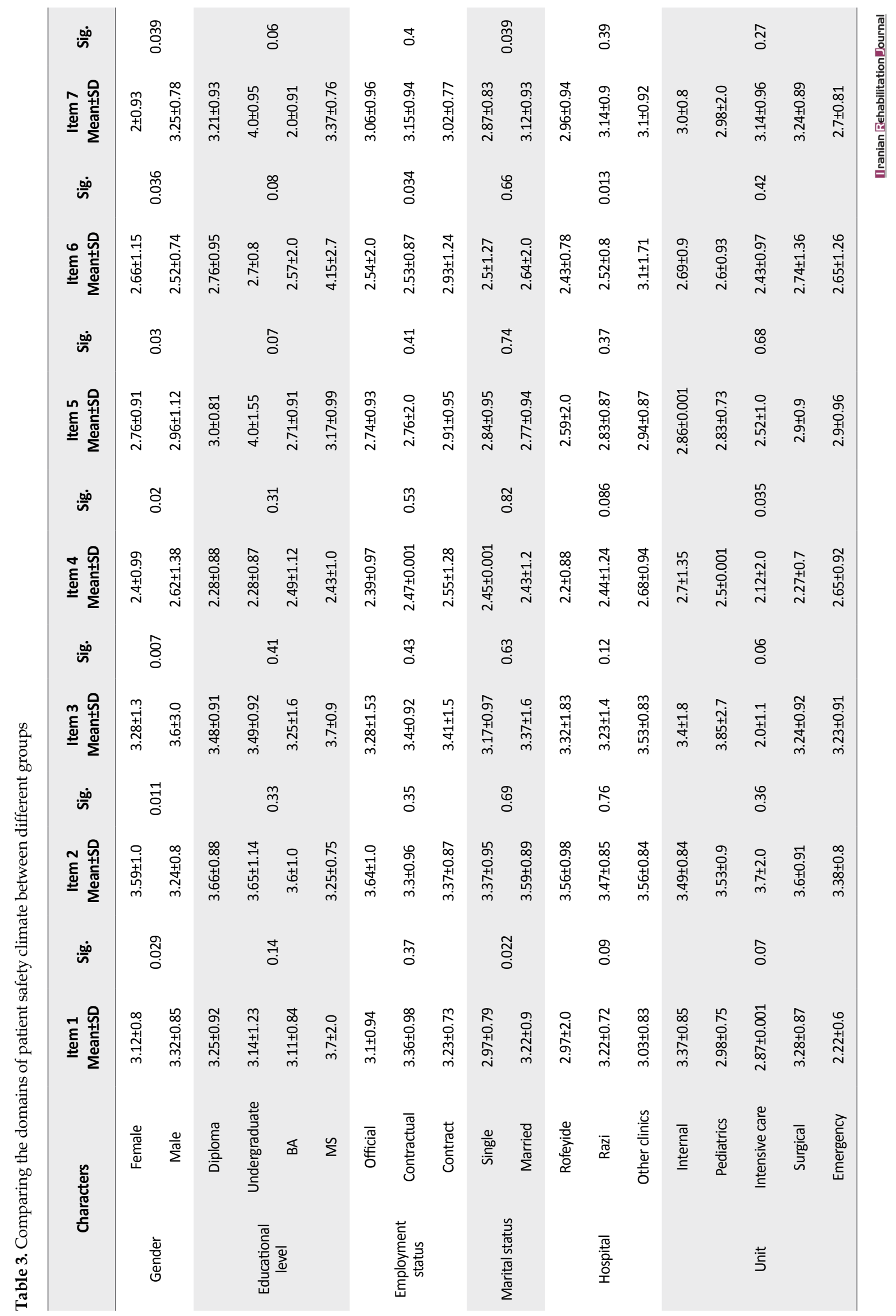


staff. This finding recommends that the higher levels of risk and complexity, and faster-paced work environment in emergency departments require relatively more consideration to safety issues than other wards [22].

Experience of healthcare staff in a particular ward, type of task, work experience and daily work hours made significant differences in patient safety [23]. Among the 7 items of questionnaire, fatigue reduction differed based on the ward. The internal ward reached the highest score $(\mathrm{P}=0.035,2.7)$. In other words, nurses in internal ward were more aware of their fatigue state and got more time to rest. Meanwhile, the Intensive Care Unit (ICU) had the lowest score (2.12) that probably was due to the higher levels of workload among staff. In addition, married nurses reported more problems than singles $(\mathrm{P}=0.039,3.12$ vs. 2.87$)$.

It can be explained by their family-related responsibilities and so, report near misses and accidents in the work. Married nurses also had higher scores in the supervisor's attitude aspect $(\mathrm{P}=0.022,3.22$ vs. 2.97).

Overall, with respect to the vital situation of nursing groups in healthcare, all factors influencing their performance and those leading to errors occurrence need to be identified and controlled. Both organizational and individual factors, such as safety climate, complexity in the organization, work schedule, stress and frustration, must be taken into consideration [24-26]. Furthermore, training healthcare staff in terms of patient safety climate and culture, as well as various structure interventions may improve patient safety and the quality of care [27].

\section{Supervisor's attitude}

The attitude of managers and supervisors is critical to have high levels of safety for both patients and staff. Snijders et al. found that reporting behavior in the neoNatal Intensive Care Unit (NICU) could be promoted by the management support of patient safety, a nonpunitive manner towards mistakes, and the perception of patient safety [28].

\section{Communication between nurses}

Good communication can highly affect the process of patient care and improve patient safety. Open communication should be established among healthcare system personnel to prevent errors by the means of the team work ability of supervisors [29].

\section{Communication with physicians}

Patient care can be improved through appropriate professional relationships. The mutual effect can be observed among the staff due to the dynamic process of relationships. Professional relationships are considered to be important factors to prevent risk; thus, communication plays a principal role in medical practice, which is necessary for patient safety [30].

\section{Fatigue reduction}

Sufficient sleep and rest as well as healthy entertainments are necessary. There was a correlation between sleep deficiency and working at home and having the second job, shift working and excessive working hours [31-35].

\section{Nursing condition}

Importance of suitable proportion between a number of patients, healthcare workers, and nurses' workload to provide adequate time to perform duties is obvious. An insufficient number of workforce in hospitals could lead to a heavy workload in healthcare staff [36]. Inadequate time to manage the patient significantly influenced the performance of hospital personnel [37]

\section{Opportunities for nursing education}

The purpose of educational opportunities is updating clinical information and be aware of changes in healthcare workers' problems and create opportunities to improve their skills. Quality management is effective in determining the educational needs of healthcare workers. In a hospital where total quality management is performed, healthcare workers make efforts to upgrade their knowledge by learning new training techniques and try to succeed in providing healthcare $[38,39]$.

\section{Reporting}

This part refers to the continuous and accurate reporting of employee's activities and occurred errors. Reasons for the lack of reporting errors were the lack of positive feedback from healthcare staff superiors, focusing on malpractice, lack of other possible factors involved in causing the error (management factors), and fear of the legal issues (fear of the consequences of reporting) [40].

\section{Conclusion}

Patient safety climate in studied hospitals was not appropriate. Considering the fact that safety climate is a major indicator of safety performance and recognizing 
its affecting factors; the assessment of situation can be effective in reducing the incidence of errors.

The studied centers need to consider patient safety climate in the daily functioning of the organizations and the routines of individuals. It is recommended to improve nurses' attitude through hospital managers' efforts to increase patient safety. Attention to personnel training is important in patient safety. In spite of the high levels of academic education among the studied subjects, patient safety climate was not desirable. Therefore, it is recommended to provide them safety training courses. However, improving culture is a time-consuming process.

\section{Ethical Considerations}

\section{Compliance with ethical guidelines}

This study was according to general ethical guidelines, and participants were assured of the confidentiality of their information.

\section{Funding}

This research did not receive any specific grant from funding agencies in the public, commercial, or not-forprofit sectors.

\section{Authors contributions}

All authors contributed in preparing this article.

\section{Conflict of interest}

The authors declare no conflict of interest.

\section{Acknowledgements}

We appreciate all the authorities of centers (Rofeyide hospital, Razi hospital, and other clinics) that helped us to conduct this study.

\section{References}

[1] Jafarjalal E, Jafarpour H, Dehghan Nayeri N, Haghani H. [Relationship between perceptions of organizational culture with Patients' safety behavior among nurses in Babol (Persian)]. Hayat. 2013; 19(3):5-16.

[2] Groves PS, Meisenbach RJ, Scott-Cawiezell J. Keeping patients safe in healthcare organizations: A structuration theory of safety culture. Journal of Advanced Nursing. 1846 67(8):1846-55. [DOI:10.1111/j.1365-2648.2011.05619.x] [PMID]
[3] Kudo Y, Kido S, Taruzuka Shahzad M, Saegusa Y, Satoh T, Aizawa Y. Safety climate and motivation toward patient safety among Japanese nurses in hospitals of fewer than 250 beds. Industrial Health. 2009; 47(1):70-9. [DOI:10.2486/indhealth.47.70] [PMID]

[4] Khandan M, Koohpaei A, Babaki F. [The effect of individual and organizational variables on Patient Safety Culture (PSC): A case study on nurses (Persian)]. Archives of Hygiene Sciences. 2016; 5(3):179-83.

[5] Energy Logistics International. A tool to assess aspects of an organisations health \& safety climate. Via Lago D'Iseo, Metropolitan: Energy Logistics International; 1997.

[6] Arefian NM, Seddighi A, Seddighi AS, Nobahar MR. Depression in the nurses of the special wards versus. Iranian Journal of Cancer Prevention. 2012; 2(3):151-4.

[7] Poursadeghiyan M, Moghimian M, Amjad R, Baneshi M, Yari A, Noroozi M, et al. Effects on job stress on Iranian clinical nurses. Annals of Tropical Medicine and Public Health. 2017; 10(4):985-8.

[8] Shahbazi A, Rahmani N, Abbasi M, Amjad RN, Marioryad $\mathrm{H}$, Khammar A, et al. Association between occupational stress and risk factors of cardiovascular disease in locomotive operators. Iranian Heart Journal. 2018; 19(2):20-6.

[9] Poursadeghiyan M, Abbasi M, Mehri A, Hami M, Raei M, Ebrahimi H. Relationship between job stress and anxiety, depression and job satisfaction in nurses in Iran. The Social Sciences. 2016; 11(9):2349-55.

[10] Zamanian Z, Zakian S, Jamali M, Kouhnavard B. [Relationship between safety culture and job stress among the Personnel of Iran telecom companies (Persian)]. Safety Promotion and Injury Prevention. 2017; 4(3):161-6.

[11] Edmondson A. Psychological safety and learning behavior in work teams. Administrative Science Quarterly. 1999; 44(2):350-83. [DOI:10.2307/2666999]

[12] Brasaite I, Kaunonen M, Martinkenas A, Suominen T. Health care professionals' attitudes regarding patient safety: cross-sectional survey. BMC Research Notes. 2016; 9:177. [DOI:10.1186/s13104-016-1977-7] [PMID] [PMCID]

[13] Li AT. Teamwork climate and patient safety attitudes: Associations among nurses and comparison with physicians in Taiwan. Journal of Nursing Care Quality. 2013; 28(1):60-7. [DOI:10.1097/NCQ.0b013e318262ac45] [PMID]

[14] Sabahi beedgoli M, Shahri S, Kebriaee A, Seyedi HR, Sarafraz Z. [Patient safety climate in medical centers of Kashan (Persian)]. Journal of Health Promotion Management. 2012; 1(1):62-72.

[15] Bonner AF, Castle NG, Men A, Handler SM. Certified nursing assistants' perceptions of nursing home patient safety culture: Is there a relationship to clinical outcomes? Journal of the American Medical Directors Association. 2009; 10(1):1120. [DOI:10.1016/j.jamda.2008.06.004] [PMID]

[16] Baghaee R, Nourani D, Khalkhali H, Pirnejad H. [Evaluating patient safety culture in personnel of academic hospitals in Urmia university of medical sciences in 2011 (Persian)]. Journal of Nursing and Midwifery Urmia University of Medical Sciences. 2012; 10(2):155-64. 
[17] Cooper AJ, Mendonca JD. A prospective study of patient assaults on nursing staff in a psychogeriatric unit. The Canadian Journal of Psychiatry. 1989; 34(5):399-404. [DOI:10.1177/ 070674378903400507]

[18] Singer S, Lin S, Falwell A, Gaba D, Baker L. Relationship of safety climate and safety performance in hospitals. Health Services Research. 2009; 44(2Pt1):399-421. [DOI:10.1111/ j.1475-6773.2008.00918.x] [PMID] [PMCID]

[19] Colla JB, Bracken AC, Kinney LM, Weeks WB. Measuring patient safety climate: A review of surveys. Archive of Quality \& Safety in Health Care. 2005; 14(5):364-6. [DOI:10.1136/ qshc.2005.014217] [PMID] [PMCID]

[20] Vifladt A, Simonsen BO, Lydersen S, Farup PG. Changes in patient safety culture after restructuring of intensive care units: Two cross-sectional studies. Intensive and Critical Care Nursing. 2016; 32:58-65. [DOI:10.1016/j.iccn.2015.06.004] [PMID]

[21] Huang DT, Clermont G, Sexton JB, Karlo CA, Miller RG, Weissfeld LA, et al. Perceptions of safety culture vary across the intensive care units of a single institution. Critical Care Medicine. 2007; 35(1):165-76. [DOI:10.1097/01. CCM.0000251505.76026.CF] [PMID]

[22] Singer SJ, Gaba DM, Falwell A, Lin S, Hayes J, Baker L. Patient safety climate in 92 US hospitals: Differences by work area and discipline. Medical Care. 2009; 47(1):23-31. [DOI:10.1097/MLR.0b013e31817e189d] [PMID]

[23] Moghery J. [Accreditation of questionnaire of patient safety culture from health care ersonnle viewpoint (Persian)]. Tehran: Tehran University of Medical Sciences; 2010.

[24] Khammar A, Amjad RN, Rohani M, Yari A, Noroozi M, Poursadeghian A, Hami M, Poursadeghiyan M. et al. Survey of shift work disorders and occupational stress among nurses: A cross-sectional study. Annals of Tropical Medicine and Public Health. 2017; 10(4):978-84.

[25] Khandan M, Vosoughi S, Maghsoudipour M. Evaluation of safety climate factors-a macroergonomics approach: A case study in Iran. Iranian Rehabilitation Journal. 2012; 10:43-6.

[26] Khandan M, Roshan Zamir S, Maghsoudipour M. [Survey of workload and job satisfaction relationship in a productive company (Persian). Iran Occupational Health Journal. 2012; 9(1):30-6.

[27] Aranaz JM, Agra Y. [The culture of patient safety: from past to future in four stages (Spanish)]. Medical Clinic Journal. 2010; 135:1-2. [DOI:10.1016/S0025-7753(10)70013-0] [PMID]

[28] Snijders C, Kollen BJ, van Lingen RA, Fetter WP, Molendijk $\mathrm{H}$. Which aspects of safety culture predict incident reporting behavior in neonatal intensive care units? A multilevel analysis. Critical Care Medicine. 2009; 37(1):61-7. [DOI:10.1097/ CCM.0b013e31819300e4] [PMID]

[29] Matsubara S, Hagihara A, Nobutomo K. Development of a patient safety climate scale in Japan. International Journal for Quality in Health Care. 2008; 20(3):211-20. [DOI:10.1093/ intqhe/mzn003] [PMID]

[30] Hemmati M, Sheikhbaglu M, Baghaie R. [Relationship between the communication skill of nurse-patient with patient safety in the critical care units (Persian)]. Journal of Clinical Nursing and Midwifery. 2014; 3(2):77-84.
[31] Abbasi M, Farhang Dehghan S, Fallah Madvari R, Mehri A, Ebrahimi MH, Poursadeghiyan M, et al. Interactive effect of background variables and workload parameters on the quality of life among nurses working in highly complex hospital units: A cross-sectional study. Journal of Clinical and Diagnostic Research. 2019; 13(1):LC08-LC13

[32] Azadboni ZD, Talarposhti RJ, Ghaljahi M, Mehri A Aarabi S, Poursadeghiyan M, et al. Effect of occupational noise exposure on sleep among workers of textile industry. Journal of Clinical \& Diagnostic Research. 2018; 12(3):18-21. [DOI:10.7860/JCDR/2018/26084.11334]

[33] Khammar A, Dalvand S, Hashemian AH, Poursadeghiyan M, Yarmohammadi S, Babakhani J, et al. Data for the prevalence of nurses' burnout in Iran (A meta-analysis dataset). Data in Brief. 2018; 20:1779-86. [DOI:10.1016/j. dib.2018.09.022] [PMID] [PMCID]

[34] Khandan M, Eyni Z, Manesh L, Khosravi Z, Biglari H, Koohpaei A. Relationship between musculoskeletal disorders and job performance among nurses and nursing aides in main educational hospital in Qom province, 2014. Research Journal of Medical Sciences. 2016; 10(4):307-12. [DOI:10.3923/ rjmsci.2016.307.312]

[35] Poursadeghiyan M, Omidi L, Hami M, Raei M, Biglari H. Drowsiness and its relation with individual characteristics among night workers in a desert hospital in Iran. International Journal of Tropical Medicine. 2016; 11:98-101. [DOI:10.3923/ ijtmed.2016.98.101]

[36] Sanders J, Cook G. ABC of patient safety. Oxford: Black well; 2007.

[37] Carayon P, Gurses AP. A human factors engineering conceptual framework of nursing workload and patient safety in intensive care units. Intensive and Critical Care Nursing 2005; 21(5):284-301. [DOI:10.1016/j.iccn.2004.12.003] [PMID]

[38] Abbatt FR. Teaching for better learning: A guide for teachers of primary health care staff. Geneva: World Health Organization; 1992.

[39] Karimpour Vazifehkhorani A, Karimzadeh M, Poursadeghiyan M, Rahmati-Najarkolaei F. Psychoeducation onimproving mental health literacy and adjustment to illness in patients with type 2 diabetes: An experimental study. Iranian Rehabilitation Journal. 2018; 16(4):395-404. [DOI:10.32598/ irj.16.4.395]

[40] Salavati S, Hatamvand F, Tabesh H. [Nurses' perspectives on causes of medication errors and non-reporting at ED (Persian)]. Iran Journal of Nursing. 2012; 25(79):72-83. 
This Page Intentionally Left Blank 